\title{
HOLOMORPHIC CONTRACTIBILITY AND OTHER PROPERTIES OF THE WEIL-PETERSSON AND VMOA TEICHMÜLLER SPACES
}

\author{
Jinhua Fan* and Jun $\mathrm{Hu}^{\dagger}$ \\ Nanjing University of Science and Technology, Department of Applied Mathematics \\ Nanjing 210094, P. R. China; jinhuafan@hotmail.com \\ Brooklyn College of CUNY, Department of Mathematics \\ Brooklyn, NY 11210, U.S.A.; junhu@brooklyn.cuny.edu \\ and Graduate Center of CUNY, Ph.D. Program in Mathematics \\ 365 Fifth Avenue, New York, NY 10016, U.S.A.; JHu1@gc.cuny.edu
}

\begin{abstract}
The Weil-Petersson and VMOA Teichmüller spaces, subspaces of the universal Teichmüller space, are complex Banach manifolds modelled on different Banach spaces. We show that they are holomorphically contractible. It is given in [28] that the Kobayashi and Teichmüller metrics coincide with each other on the Weil-Petersson Teichmüller space. We show that this property is also true on the VMOA Teichmüller space. A couple of other properties of the two spaces are also obtained.
\end{abstract}

\section{Introduction}

Let $L^{\infty}(\mathbf{D})$ denote the Banach space of essentially bounded measurable complexvalued functions defined on the open unit disk $\mathbf{D}$, and let $M(\mathbf{D})$ be the open unit ball in $L^{\infty}(\mathbf{D})$ centered at the 0 -constant function. For each element $\mu \in M(\mathbf{D})$, there is a unique quasiconformal homeomorphism $f^{\mu}$ of $\mathbf{D}$ with Beltrami coefficient $\mu$ and normalized to fix three points $1,-1$ and $i$. Two elements $\mu$ and $\nu$ of $M(\mathbf{D})$ are equivalent if $\left.f^{\mu}\right|_{\mathbf{S}^{1}}=\left.f^{\nu}\right|_{\mathbf{S}^{1}}$, where $\mathbf{S}^{1}=\partial \mathbf{D}$. Denote by $[\mu]$ or $\left[f^{\mu}\right],\left[\left.f^{\mu}\right|_{\mathbf{S}^{1}}\right]$ the equivalent class of $\mu$. The universal Teichmüller space $T(\mathbf{D})$ can be defined as

$$
T(\mathbf{D})=\{[\mu]: \mu \in M(\mathbf{D})\} \text {. }
$$

A natural projection from $M(\mathbf{D})$ to $T(\mathbf{D})$ is defined by

$$
\Phi: M(\mathbf{D}) \rightarrow T(\mathbf{D}): \mu \mapsto[\mu] .
$$

Let $\widehat{\mathbf{C}}$ be the extended complex plane and $\mathbf{D}^{*}=\widehat{\mathbf{C}} \backslash \overline{\mathbf{D}}$. Given each element $\mu \in M(\mathbf{D})$, let $f_{\mu}$ be the quasiconformal mapping on $\widehat{\mathbf{C}}$ with Beltrami coefficient $\mu$ on $\mathbf{D}$ and 0 on $\mathbf{D}^{*}$ and normalized on $\mathbf{D}^{*}$ as

$$
f_{\mu}(z)=z+\frac{b_{1}}{z}+\frac{b_{2}}{z^{2}} \cdots
$$

Then $\left.f_{\mu}\right|_{\mathbf{D}^{*}}=\left.f_{\nu}\right|_{\mathbf{D}^{*}}$ if and only if $\left.f^{\mu}\right|_{\mathbf{S}^{1}}=\left.f^{\nu}\right|_{\mathbf{S}^{1}}$.

Let $S_{\left.f_{\mu}\right|_{\mathbf{D}^{*}}}$ be the Schwarzian derivative of $\left.f_{\mu}\right|_{\mathbf{D}^{*}}$ and let $B\left(\mathbf{D}^{*}\right)$ be the Banach space of holomorphic quadratic differentials $\phi$ on $\mathbf{D}^{*}$ under the following norm

$$
\|\phi\|_{B}=\sup _{z \in \mathbf{D}^{*}}|\phi(z)| \rho_{\mathbf{D}^{*}}^{-2}(z)<\infty,
$$

doi:10.5186/aasfm.2016.4137

2010 Mathematics Subject Classification: Primary 30C75, 30F60.

Key words: Kobayashi metric, Weil-Petersson Teichmüller space, VMOA Teichmüller space, holomorphic contractibility.

*This work is supported by NNSF of China (No. 11201228 and No. 11571172).

$\dagger$ The work is partially supported by PSC-CUNY grants. 
where $\rho_{\mathbf{D}^{*}}(z)=1 /\left(|z|^{2}-1\right)$ is the Poincaré density on $\mathbf{D}^{*}$. Using the Bers embedding

$$
\beta: T(\mathbf{D}) \rightarrow B\left(\mathbf{D}^{*}\right): \beta([\mu])=S_{\left.f_{\mu}\right|_{\mathbf{D}^{*}}},
$$

one can introduce a complex structure on $T(\mathbf{D})$ such that $\Phi$ is a holomorphic split submersion.

There are several interesting subspaces of $T(\mathbf{D})$, which are also complex Banach manifolds but modelled on Banach spaces with different norms. This paper involves three of them: the space $T_{0}(\mathbf{D})$, the Weil-Petersson Teichmüller space and the VMOA Teichmüller space. In the following, we first give a brief introduction to these three spaces.

An element $\mu \in M(\mathbf{D})$ is called a vanishing Beltrami coefficient if for any $\epsilon>0$, there exist $0<r<1$ such that $\left\|\left.\mu\right|_{\mathbf{D} \backslash \mathbf{D}_{r}}\right\|_{\infty}<\epsilon$, where $\mathbf{D}_{r}=\{z:|z|<r\}$. Let $M_{0}(\mathbf{D})$ be the collection of all vanishing Beltrami coefficients. Then $T_{0}(\mathbf{D})$ is defined to be

$$
T_{0}(\mathbf{D})=\left\{[\mu]: \mu \in M_{0}(\mathbf{D})\right\} .
$$

The space $T_{0}(\mathbf{D})$ is a normal subgroup of $T(\mathbf{D})$ under the composition [14], and the quotient space $T(\mathbf{D}) / T_{0}(\mathbf{D})$ is called the asymptotic Teichmüller space of $\mathbf{D}$ and denoted by $A T(\mathbf{D})$. For studies of $A T(\mathbf{D})$, we refer to [14], [7], [8] and [10]. Let $B_{0}\left(\mathbf{D}^{*}\right)$ be the subset of $B\left(\mathbf{D}^{*}\right)$ consisting of all $\phi \in B\left(\mathbf{D}^{*}\right)$ such that

$$
\lim _{r \rightarrow 1^{+}} \sup _{|z|=r}|\phi(z)| \rho_{\mathbf{D}^{*}}^{-2}(z)=0 .
$$

Then $[\mu] \in T_{0}(\mathbf{D})$ if and only if $\beta([\mu]) \in B_{0}\left(\mathbf{D}^{*}\right)[10]$, and $T_{0}(\mathbf{D})$ is a complex Banach manifold modelled on the Banach space $\left(B_{0}\left(\mathbf{D}^{*}\right),\|\cdot\|_{B}\right)$. Furthermore, the inclusion map

$$
i: T_{0}(\mathbf{D}) \rightarrow T(\mathbf{D})
$$

is holomorphic.

Let $M_{2}(\mathbf{D})$ be the subset of $M(\mathbf{D})$ consisting of all $\mu \in M(\mathbf{D})$ such that

$$
\int_{\mathbf{D}}|\mu(z)|^{2} \rho_{\mathbf{D}}^{2}(z) d x d y<\infty
$$

where $\rho_{\mathbf{D}}(z)=1 /\left(1-|z|^{2}\right)$ is the Poincaré density on $\mathbf{D}$. (For a convenience of using fewer symbols in the expressions of integrals in the next section, we consider $\rho_{\mathbf{D}}(z)=0$ if $|z| \geq 1$.) The space $T_{2}(\mathbf{D})$ is defined as

$$
T_{2}(\mathbf{D})=\left\{[\mu]: \mu \in M_{2}(\mathbf{D})\right\},
$$

which is called the integrable Teichmüller space in [3] or the Weil-Petersson Teichmüller space in [22]. Let $B_{2}\left(\mathbf{D}^{*}\right)$ be the subset of $B\left(\mathbf{D}^{*}\right)$ consisting of all $\phi \in B\left(\mathbf{D}^{*}\right)$ such that

$$
\|\phi\|_{2}=\left\{\int_{\mathbf{D}^{*}}|\phi(z)|^{2} \rho_{\mathbf{D}^{*}}^{-2}(z) d x d y\right\}^{\frac{1}{2}}<\infty .
$$

It was proved in [3] that $[\mu] \in T_{2}(\mathbf{D})$ if and only if $\beta([\mu]) \in B_{2}\left(\mathbf{D}^{*}\right)$, and $T_{2}(\mathbf{D})$ is a complex Banach manifold modelled on the Banach space $\left(B_{2}\left(\mathbf{D}^{*}\right),\|\cdot\|_{2}\right)$. It is also proved in Lemma 2 of [3] that (1.2) implies (1.1). Therefore,

$$
T_{2}(\mathbf{D}) \subset T_{0}(\mathbf{D}) \subset T(\mathbf{D}) .
$$

Furthermore, $T_{2}(\mathbf{D})$ is a group under the composition [3]. For more studies of $T_{2}(\mathbf{D})$, we refer to [16], [24] and [26]. 
A positive measure $\lambda$ defined on a simply connected domain $\Omega$ of $\widehat{\mathbf{C}}$ is called a Carleson measure if

$$
\|\lambda\|_{c}^{2}=\sup _{z, r}\left\{\frac{\lambda(\Omega \cap U(z, r))}{r}: z \in \partial \Omega, 0<r<\operatorname{diameter}(\Omega)\right\}<\infty,
$$

where $U(z, r)=\{w:|w-z|<r\}$. A Carleson measure $\lambda$ is said to be vanishing if

$$
\lim _{r \rightarrow 0} \frac{\lambda(\Omega \cap U(z, r))}{r}=0
$$

uniformly on $z \in \partial \Omega$. We denote by $C M_{0}(\Omega)$ the set of all vanishing Carleson measures on $\Omega$. Let $M_{v}(\mathbf{D})$ be the subset of $M(\mathbf{D})$ consisting of all $\mu \in M(\mathbf{D})$ such that $|\mu(z)|^{2} \rho_{\mathbf{D}}(z) \in C M_{0}(\mathbf{D})$. Then the VMOA Teichmüller space $T_{v}(\mathbf{D})$ is defined by

$$
T_{v}(\mathbf{D})=\left\{[\mu]: \mu \in M_{v}(\mathbf{D})\right\} .
$$

Let $B_{v}\left(\mathbf{D}^{*}\right)$ be the subset of $B\left(\mathbf{D}^{*}\right)$ consisting of all $\phi \in B\left(\mathbf{D}^{*}\right)$ such that

$$
|\phi(z)|^{2} \rho_{\mathbf{D}^{*}}^{-3}(z) \in C M_{0}\left(\mathbf{D}^{*}\right),
$$

which is a Banach space under the norm

$$
\|\phi\|_{v}=\left\||\phi|^{2} \rho_{\mathbf{D}^{*}}^{-3}\right\|_{c} .
$$

It was proved in [23] that $[\mu] \in T_{v}(\mathbf{D})$ if and only if $\beta([\mu]) \in B_{v}\left(\mathbf{D}^{*}\right)$, and $T_{v}(\mathbf{D})$ is a Banach complex manifold modelled on $\left(B_{v}\left(\mathbf{D}^{*}\right),\|\cdot\|_{v}\right)$.

The proof of Lemma 2 in [3] can be modified to show that (1.4) implies (1.1) [23]. Therefore,

$$
T_{v}(\mathbf{D}) \subset T_{0}(\mathbf{D}) \subset T(\mathbf{D}) .
$$

Furthermore, $T_{v}(\mathbf{D})$ is also a group under the composition [23]. For more studies of $T_{v}(\mathbf{D})$, we refer to [1], [2] and [20].

Let $\mathcal{T}\left(\mathbf{D}^{*}\right)=\beta(T(\mathbf{D})), \mathcal{T}_{2}\left(\mathbf{D}^{*}\right)=\beta\left(T_{2}(\mathbf{D})\right)$ and $\mathcal{T}_{v}\left(\mathbf{D}^{*}\right)=\beta\left(T_{v}(\mathbf{D})\right)$. Then

$$
\mathcal{T}\left(\mathbf{D}^{*}\right) \subset B\left(\mathbf{D}^{*}\right), \quad \mathcal{T}_{2}\left(\mathbf{D}^{*}\right) \subset B_{2}\left(\mathbf{D}^{*}\right) \quad \text { and } \quad \mathcal{T}_{v}\left(\mathbf{D}^{*}\right) \subset B_{v}\left(\mathbf{D}^{*}\right)
$$

Furthermore,

$$
\mathcal{T}_{2}\left(\mathbf{D}^{*}\right) \subset \mathcal{T}\left(\mathbf{D}^{*}\right) \quad \text { and } \quad \mathcal{T}_{v}\left(\mathbf{D}^{*}\right) \subset \mathcal{T}\left(\mathbf{D}^{*}\right)
$$

These subspaces of $T(\mathbf{D})$ are introduced for different purposes: $T_{0}(\mathbf{D})$ is used to define the asymptotic Teichmüller space of $\mathbf{D} ; T_{2}(\mathbf{D})$ plays an important role in the study of the Weil-Petersson geometry of the universal Teichmüller space $[16,22,26]$; $T_{v}(\mathbf{D})$ has applications in harmonic analysis (see [1], [23] and references therein). In this paper, we study complex properties of $T_{2}(\mathbf{D})$ and $T_{v}(\mathbf{D})$.

Note that $T_{2}(\mathbf{D})$ (resp. $\left.T_{v}(\mathbf{D})\right)$ and $T(\mathbf{D})$ are complex Banach manifolds modelled on Banach spaces with different norms. In fact, the topology induced by $\|\cdot\|_{2}$ (resp. $\|\cdot\|_{v}$ ) on $T_{2}(\mathbf{D})$ (resp. $T_{v}(\mathbf{D})$ ) is stronger than the one induced by $\|\cdot\|_{B}$ restricted on $T_{2}(\mathbf{D})$ (resp. $\left.T_{v}(\mathbf{D})\right)([3],[23])$. Therefore, it is not obvious that the inclusion map from $\mathcal{T}_{2}\left(\mathbf{D}^{*}\right)$ (resp. $\mathcal{T}_{v}\left(\mathbf{D}^{*}\right)$ ) to $\mathcal{T}\left(\mathbf{D}^{*}\right)$ is holomorphic. In this paper, we first give a proof to the following folklore theorem.

Theorem 1. (Folklore Theorem) The inclusion maps

$$
i_{2}:\left(\mathcal{T}_{2}\left(\mathbf{D}^{*}\right),\|\cdot\|_{2}\right) \rightarrow\left(\mathcal{T}\left(\mathbf{D}^{*}\right),\|\cdot\|_{B}\right)
$$

and

are holomorphic.

$$
i_{v}:\left(\mathcal{T}_{v}\left(\mathbf{D}^{*}\right),\|\cdot\|_{v}\right) \rightarrow\left(\mathcal{T}\left(\mathbf{D}^{*}\right),\|\cdot\|_{B}\right)
$$


A complex manifold $X$ is said to be holomorphic contractible to a point $x_{0} \in X$ if there is a continuous map

$$
F:[0,1] \times X \rightarrow X
$$

such that for each $x \in X, F(0, x)=x$ and $F(1, x)=x_{0}$, and for each $t \in[0,1]$, the map $x \mapsto F(t, x)$ is holomorphic from $X$ to itself and fixes the point $x_{0}$. Such a map $F$ is called a holomorphic contraction of $X$ to $x_{0}$.

It was shown respectively in [5], [3] and [27] that Teichmüller spaces, the spaces $\mathcal{T}_{2}(\mathbf{D})$ and $\mathcal{T}_{v}(\mathbf{D})$ are contractible. Since Teichmüller spaces are complex Banach manifolds, an interesting question is to ask whether or not their images under the Bers embedding $\beta$ are holomorphically contractible. It is still an open question whether or not $\mathcal{T}\left(\mathbf{D}^{*}\right)$ is holomorphically contractible, but Earle proved in [6] that $\mathcal{T}_{0}\left(\mathbf{D}^{*}\right)$ is holomorphically contracted to the base point. The main result of this paper is to show that $\mathcal{T}_{2}\left(\mathbf{D}^{*}\right)$ and $\mathcal{T}_{v}\left(\mathbf{D}^{*}\right)$ are also holomorphically contracted to the base point.

Theorem 2. (Main Theorem) Both $\mathcal{T}_{2}\left(\mathbf{D}^{*}\right)$ and $\mathcal{T}_{v}\left(\mathbf{D}^{*}\right)$ are holomorphically contracted to the base point $\beta([0])$.

The Kobayashi pseudo metric is defined on any complex Banach manifold, and hence on any Teichmüller space. Then one likes to study whether or not the Kobayashi pseudo metric and the Teichmüller metric coincide with each other on a Teichmüller space. This is proved to be true by Royden on finite-dimensional Teichmüller spaces [21] and by Gardiner on infinite-dimensional Teichmüller spaces [12]. A unified proof of the coincidence of the two metrics on all Teichmüller spaces is given in [9]. The same problem is studied on the space $T_{0}(\mathbf{D})$. It is proved in [8] that the Teichmüller metric and the Kobayashi metric are equal on $T_{0}(\mathbf{D})$, see [15] for a direct proof. The coincidence of the Kobayashi metric and the Teichmüller metric on $T_{2}(\mathbf{D})$ is given as Theorem 1.1 in [28]. In this paper, we show that this property also holds on $T_{v}(\mathbf{D})$.

Theorem 3. The Kobayashi metric coincides with the Teichmüller metric on $T_{v}(\mathbf{D})$.

By an invariant metric $d_{X}$ on a complex Banach manifold $X$ we mean a pseudo metric that satisfies

$$
d_{\mathbf{D}}\left(f\left(x_{1}\right), f\left(x_{2}\right)\right) \leq d_{X}\left(x_{1}, x_{2}\right) \quad \text { and } \quad d_{X}\left(g\left(z_{1}\right), g\left(z_{2}\right)\right) \leq d_{\mathbf{D}}\left(z_{1}, z_{2}\right)
$$

for any holomorphic functions $f: X \rightarrow \mathbf{D}$ and $g: \mathbf{D} \rightarrow X$ and two points $x_{1}, x_{2} \in X$ or $z_{1}, z_{2} \in \mathbf{D}$. It is proved in [18] that any invariant metric on any asymptotic Teichmüller space is a complete metric. As an application of our Theorems 1 and 3, we obtain the following property.

Corollary 1. Any invariant metric on $T_{2}(\mathbf{D})$ or $T_{v}(\mathbf{D})$ is not complete.

The Kobayashi metric is an invariant metric. Theorem 1.1 of [28], our Theorem 3 and the previous corollary imply the following corollary.

Corollary 2. Both $T_{2}(\mathbf{D})$ and $T_{v}(\mathbf{D})$ are not closed in $T(\mathbf{D})$ under the Teichmüller metric.

Note that $T_{0}(\mathbf{D})$ is complete and closed in $T(\mathbf{D})$ under the Teichmüller metric and any invariant metric on $T_{0}(\mathbf{D})$ is complete.

The paper is organized as follows. We prove Theorems 1 and 2 in Section 2 and Theorem 3 and Corollary 1 in Section 3. 
Acknowledgement. Both authors wish to thank the referees for their comments, suggestions and corrections of typos.

\section{Proofs of Theorems 1 and 2}

Let $X$ and $Y$ be two complex Banach spaces, and $U$ be a non-empty open subset in $X$. A mapping $f: U \rightarrow Y$ is said to be holomorphic if $f$ is locally bounded and the complex Gateaux derivative $d_{u} f(x)$ defined by

$$
d_{u} f(x)=\lim _{t \rightarrow 0} \frac{f(u+t x)-f(u)}{t}
$$

exists for each $(u, x) \in(U, X)$. There are various equivalent definitions of holomorphy ([19], [24]). In this paper, we use the following characterization of holomorphy ([19], [23]): a continuous map $f: U \rightarrow Y$ is holomorphic if for each $(u, x) \in(U, X)$ and each element $y^{*}$ from a total subset $Y_{0}^{*}$ of the dual space $Y^{*}, y^{*}(f(u+t x))$ is a holomorphic function in a neighborhood of zero in the complex plane. Here a subset $Y_{0}^{*}$ of $Y^{*}$ is total if $y^{*}(y)=0$ for all $y^{*} \in Y_{0}^{*}$ implies $y=0$.

In order to prove Theorem 1, we need the following two known results.

Lemma 1. [3] There exists a constant $C>0$ such that

$$
\|\phi\|_{B} \leq C\|\phi\|_{2} \text {. }
$$

Lemma 2. [23] There exists a constant $C>0$ such that

$$
\|\phi\|_{B} \leq C\|\phi\|_{v}
$$

Proof of Theorem 1. By Lemma 1, we know that $i_{2}$ is continuous. For each $z \in \mathbf{D}^{*}$ and $\phi \in B\left(\mathbf{D}^{*}\right)$, we define $l_{z}(\phi)=\phi(z)$. Then $|\phi(z)| \leq\|\phi\|_{B}\left(|z|^{2}-1\right)^{-2}$, which implies $\left\|l_{z}\right\| \leq\left(|z|^{2}-1\right)^{-2}$. Thus $l_{z} \in B^{*}\left(\mathbf{D}^{*}\right)$. Set $A=\left\{l_{z}: z \in \mathbf{D}^{*}\right\}$, it is obviously $A$ is a total subset of the dual space $B^{*}\left(\mathbf{D}^{*}\right)$. Now for each pair $(\phi, \varphi) \in\left(\mathcal{T}_{2}\left(\mathbf{D}^{*}\right), B_{2}\left(\mathbf{D}^{*}\right)\right)$ and any $t$ on the complex plane with $|t|$ small, it follows from Lemma 1 that $i_{2}(\phi+t \varphi) \in B\left(\mathbf{D}^{*}\right)$. Then for each fixed $z \in \mathbf{D}^{*}$, we obtain that $l_{z}\left(i_{2}(\phi+t \varphi)\right)=\phi(z)+t \varphi(z)$ is a holomorphic function of $t$. Using the characterization of holomorphy, we conclude that the inclusion map $i_{2}$ is holomorphic. Using Lemma 2 and a similar argument, we can see that $i_{v}$ is also holomorphic.

Before giving a proof of Theorem 2, let us recall some results and notation in [6]. In order to use some notation introduced in [6], we replace the four spaces $B\left(\mathbf{D}^{*}\right)$, $B_{0}\left(\mathbf{D}^{*}\right), B_{2}\left(\mathbf{D}^{*}\right)$ and $B_{v}\left(\mathbf{D}^{*}\right)$ by their counterparts defined on $\mathbf{D}$. Let $A(z)=\frac{1}{z}$ and $B(\mathbf{D})$ be the collection of holomorphic functions $\varphi$ on $\mathbf{D}$ with

$$
\|\varphi\|_{B}=\sup _{z \in \mathbf{D}}|\varphi(z)| \rho_{\mathbf{D}}^{-2}(z)<\infty .
$$

Then an isometric isomorphism $A^{*}$ from $B\left(\mathbf{D}^{*}\right)$ to $B(\mathbf{D})$ is defined by

$$
A^{*}(\phi)=(\phi \circ A)\left(A^{\prime}\right)^{2},
$$

where $\phi \in B\left(\mathbf{D}^{*}\right)$.

Let $B_{0}(\mathbf{D}), B_{2}(\mathbf{D})$ and $B_{v}(\mathbf{D})$ be the subsets of $B(\mathbf{D})$ consisting of all $\varphi \in B(\mathbf{D})$ such that

$$
\lim _{r \rightarrow 1^{-}} \sup _{|z|=r}|\varphi(z)| \rho_{\mathbf{D}}^{-2}(z)=0, \quad \int_{\mathbf{D}}|\varphi(z)|^{2} \rho_{\mathbf{D}}^{-2}(z) d x d y<\infty
$$

and

$$
|\varphi(z)|^{2} \rho_{\mathbf{D}}^{-3}(z) \in C M_{0}(\mathbf{D})
$$


respectively. Then $A^{*}\left(B_{0}\left(\mathbf{D}^{*}\right)\right)=B_{0}(\mathbf{D}), A^{*}\left(B_{2}\left(\mathbf{D}^{*}\right)\right)=B_{2}(\mathbf{D})$ and $A^{*}\left(B_{v}\left(\mathbf{D}^{*}\right)\right)=$ $B_{v}(\mathbf{D})$. We denote by $\mathcal{T}_{0}(\mathbf{D}), \mathcal{T}_{2}(\mathbf{D})$ and $\mathcal{T}_{v}(\mathbf{D})$ the images under $A^{*}$ of $\mathcal{T}_{0}\left(\mathbf{D}^{*}\right)$, $\mathcal{T}_{2}\left(\mathbf{D}^{*}\right)$ and $\mathcal{T}_{v}\left(\mathbf{D}^{*}\right)$ respectively. Then $\mathcal{T}_{2}(\mathbf{D})=\mathcal{T}_{0}(\mathbf{D}) \cap B_{2}(\mathbf{D})$ and $\mathcal{T}_{v}(\mathbf{D})=\mathcal{T}_{0}(\mathbf{D}) \cap$ $B_{v}(\mathbf{D})$. In order to conclude Theorem 2 , it suffices to prove that $\mathcal{T}_{2}(\mathbf{D})$ and $\mathcal{T}_{v}(\mathbf{D})$ are holomorphically contractible. In the remaining part of this section, the following theorem of Earle in [6] is employed to prove our Theorem 2.

Theorem A. [6] Given a point $\tau$ in the closed unit disk $\overline{\mathbf{D}}$ and $\varphi \in \mathcal{T}_{0}(\mathbf{D})$, let $g_{\tau}(z)=\tau z$ and $g_{\tau}^{*}(\varphi)=\left(\varphi \circ g_{\tau}\right)\left(g_{\tau}^{\prime}\right)^{2}$. Then

$$
G: \overline{\mathbf{D}} \times \mathcal{T}_{0}(\mathbf{D}) \longrightarrow \mathcal{T}_{0}(\mathbf{D}):(\tau, \varphi) \longmapsto g_{\tau}^{*}(\varphi)
$$

is a continuous map. Furthermore, for each fixed $\tau \in \overline{\mathbf{D}}$ the map $\varphi \mapsto G(\tau, \varphi)$ is a holomorphic map from $\mathcal{T}_{0}(\mathbf{D})$ to itself.

Proof of Theorem 2. Let $G(\tau, \varphi)$ be the map defined in Theorem A. If $G(\tau, \varphi)$ is continuous and maps holomorphically $\mathcal{T}_{2}(\mathbf{D})$ to itself for any $\tau \in[0,1]$, then the map $F(t, \varphi)=G(1-t, \varphi):[0,1] \times \mathcal{T}_{2}(\mathbf{D}) \rightarrow \mathcal{T}_{2}(\mathbf{D})$ contracts $\mathcal{T}_{2}(\mathbf{D})$ holomorphically to the base point of $\mathcal{T}_{2}(\mathbf{D})$.

In the following, by three steps, we will prove $G(\tau, \varphi)$ is continuous and maps holomorphically $\mathcal{T}_{2}(\mathbf{D})$ to itself for any $\tau \in[0,1]$.

Step 1. We prove that $G(\tau, \varphi)=\left(\varphi \circ g_{\tau}\right)\left(g_{\tau}^{\prime}\right)^{2}$ maps $\mathcal{T}_{2}(\mathbf{D})$ into itself for each $\tau \in[0,1]$. By Theorem $\mathrm{A}$ and $\mathcal{T}_{2}(\mathbf{D})=\mathcal{T}_{0}(\mathbf{D}) \cap B_{2}(\mathbf{D})$, we only need to prove $\|G(\tau, \varphi)\|_{2}<\infty$ for any $(\tau, \varphi) \in[0,1] \times \mathcal{T}_{2}(\mathbf{D})$. Let $(\tau, \varphi) \in[0,1] \times \mathcal{T}_{2}(\mathbf{D})$ and denote by $\mathbf{D}_{\tau}=\{z:|z|<\tau\}$. Then

$$
\begin{aligned}
\|G(\tau, \varphi)\|_{2}^{2} & =\int_{\mathbf{D}}|G(\tau, \varphi)|^{2} \rho_{\mathbf{D}}^{-2}(z) d x d y=\int_{\mathbf{D}} \tau^{4}|\varphi(\tau z)|^{2} \rho_{\mathbf{D}}^{-2}(z) d x d y \\
& \leq \tau^{2} \int_{\mathbf{D}} \tau^{2}|\varphi(\tau z)|^{2} \rho_{\mathbf{D}}^{-2}(\tau z) d x d y=\tau^{2} \int_{\mathbf{D}_{\tau}}|\varphi(z)|^{2} \rho_{\mathbf{D}}^{-2}(z) d x d y \\
& \leq \tau^{2} \int_{\mathbf{D}}|\varphi(z)|^{2} \rho_{\mathbf{D}}^{-2}(z) d x d y=\tau^{2}\|\varphi\|_{2}^{2}<\infty
\end{aligned}
$$

which implies $G(\tau, \varphi) \in \mathcal{T}_{2}(\mathbf{D})$.

Step 2. We prove that $G:[0,1] \times \mathcal{T}_{2}(\mathbf{D}) \rightarrow \mathcal{T}_{2}(\mathbf{D})$ is continuous. We first show $G(\tau, \varphi)$ is continuous on the variable $\varphi \in \mathcal{T}_{2}(\mathbf{D})$ for each fixed $\tau \in[0,1]$ and then show $G(\tau, \varphi)$ is continuous on the variable $\tau \in[0,1]$ for each fixed $\varphi \in \mathcal{T}_{2}(\mathbf{D})$. obtain

Let $\tau \in[0,1]$ and $\varphi, \psi \in \mathcal{T}_{2}(\mathbf{D})$. Using a similar process of steps in (2.1), we

$$
\|G(\tau, \varphi)-G(\tau, \psi)\|_{2} \leq \tau\|\varphi-\psi\|_{2}
$$

Hence $G(\tau, \varphi)$ is continuous at any point $\varphi \in \mathcal{T}_{2}(\mathbf{D})$ for each fixed $\tau \in[0,1]$.

Now we prove that $G(\tau, \varphi)$ is continuous on the variable $\tau \in[0,1]$ for each fixed $\varphi \in \mathcal{T}_{v}(\mathbf{D})$. We divide the proof into two cases.

Case 1: We assume that a sequence $\left\{\tau_{n}\right\}_{n=1}^{\infty}$ of points in $[0,1]$ converges to a point $\tau \in[0,1)$. Then for any sufficiently large $n$ and all $z \in \mathbf{D}, \tau_{n} z$ and $\tau z$ are contained in a compact subset of $\mathbf{D}$ and hence

$$
\lim _{n \rightarrow \infty} \sup _{z \in \mathbf{D}}\left|\tau_{n}^{4} \varphi^{2}\left(\tau_{n} z\right)-\tau^{4} \varphi^{2}(\tau z)\right|=0 .
$$

Thus,

$$
\left\|G\left(\tau_{n}, \varphi\right)-G(\tau, \varphi)\right\|_{2}^{2} \leq\left(\sup _{z \in \mathbf{D}}\left|\tau_{n}^{4} \varphi^{2}\left(\tau_{n} z\right)-\tau^{4} \varphi^{2}(\tau z)\right|\right) \int_{\mathbf{D}} \rho_{\mathbf{D}}^{-2}(z) d x d y \rightarrow 0
$$


as $n \rightarrow \infty$. This means that $G(\cdot, \varphi)$ is continuous at any point $\tau \in[0,1)$ for each fixed $\varphi \in \mathcal{T}_{2}(\mathbf{D})$.

Case 2: Assume that a sequence $\left\{\tau_{n}\right\}_{n=1}^{\infty}$ of points in $[0,1]$ converges to 1 . Denote by $\mathbf{D}_{r, 1}=\{z: r<|z|<1\}$. Since $\varphi \in \mathcal{T}_{2}(\mathbf{D})$, it follows that for any $\epsilon>0$, there exists $r \in(0,1)$ such that

$$
\int_{\mathbf{D}_{r, 1}}|\varphi(z)|^{2} \rho_{\mathbf{D}}^{-2}(z) d x d y<\epsilon .
$$

Let $r^{\prime} \in(r, 1)$. Since $\tau_{n}$ converges to 1 , there exists $N \in \mathbf{N}$ such that $\tau_{n} z \in \mathbf{D}_{r, 1}$ for any $n>N$ and any $z \in \mathbf{D}_{r^{\prime}, 1}$. Using a similar process of steps in (2.1), we obtain if $n>N$, then

$$
\begin{aligned}
\int_{\mathbf{D}_{r^{\prime}, 1}}\left|G\left(\tau_{n}, \varphi\right)-\varphi\right|^{2} \rho_{\mathbf{D}}^{-2}(z) d x d y & \leq \int_{\mathbf{D}_{r^{\prime}, 1}} 2\left(\left|G\left(\tau_{n}, \varphi\right)\right|^{2}+|\varphi(z)|^{2}\right) \rho_{\mathbf{D}}^{-2}(z) d x d y \\
& \leq 4 \int_{\mathbf{D}_{r, 1}}|\varphi(z)|^{2} \rho_{\mathbf{D}}^{-2}(z) d x d y<4 \epsilon
\end{aligned}
$$

Using the same argument in Case 1, we can show

$$
\lim _{n \rightarrow \infty} \int_{\mathbf{D}_{r^{\prime}}}\left|G\left(\tau_{n}, \varphi\right)-\varphi\right|^{2} \rho_{\mathbf{D}}^{-2}(z) d x d y=0 .
$$

Thus

$$
\lim _{n \rightarrow \infty}\left\|G\left(\tau_{n}, \varphi\right)-\varphi\right\|_{2}=0
$$

which means that $G(\tau, \varphi)$ is continuous at $\tau=1$ for each fixed $\varphi \in \mathcal{T}_{2}(\mathbf{D})$.

Step 3. We prove that $G(\tau, \cdot): \mathcal{T}_{2}(\mathbf{D}) \rightarrow B_{2}(\mathbf{D})$ is holomorphic for each fixed $\tau \in[0,1]$. Let $z \in \mathbf{D}$ and $\varphi \in B_{2}(\mathbf{D})$ and define $l_{z}(\varphi)=\varphi(z)$. From the proof of Theorem 1 and $B_{2}(\mathbf{D}) \subset B(\mathbf{D})$, we know $l_{z} \in B^{*}(\mathbf{D}) \subset B_{2}^{*}(\mathbf{D})$. Set $A=\left\{l_{z}: z \in\right.$ $\left.\mathbf{D}^{*}\right\}$, it is obviously $A$ is a total subset of the dual space $B_{2}^{*}(\mathbf{D})$. Now let $(\varphi, \psi) \in$ $\left(\mathcal{T}_{2}(\mathbf{D}), B_{2}(\mathbf{D})\right)$ and let $t$ be a complex variable with small $|t|$. For each fixed $z \in \mathbf{D}$ and $\tau \in[0,1], l_{z}(G(\tau, \varphi+t \psi))=\tau^{2}(\varphi(\tau z)+t \psi(\tau z))$ is a holomorphic function of $t$. Consequently, $G(\tau, \cdot): \mathcal{T}_{2}(\mathbf{D}) \rightarrow B_{2}(\mathbf{D})$ is holomorphic for each fixed $\tau \in[0,1]$.

In summary, the previous three steps imply that $G(\tau, \varphi)$ is continuous on $\tau \in[0,1]$ and $\varphi \in \mathcal{T}_{2}(\mathbf{D})$ and it maps $\mathcal{T}_{2}(\mathbf{D})$ holomorphically to itself for each fixed $\tau \in[0,1]$.

The second half of the proof is to show that $G(\tau, \varphi)$ is a continuous function defined on $[0,1] \times T_{v}(\mathbf{D})$ and it maps $\mathcal{T}_{v}(\mathbf{D})$ holomorphically to itself for each fixed $\tau \in[0,1]$. Then $F(t, \varphi)=G(1-t, \varphi):[0,1] \times \mathcal{T}_{v}(\mathbf{D}) \rightarrow \mathcal{T}_{v}(\mathbf{D})$ contracts $\mathcal{T}_{v}(\mathbf{D})$ holomorphically to the base point of $\mathcal{T}_{v}(\mathbf{D})$. We divide the proof into the following four steps.

Step $1^{\prime}$. We prove that for each $(\tau, \varphi) \in[0,1] \times \mathcal{T}_{v}(\mathbf{D}),\|G(\tau, \varphi)\|_{v}^{2} \leq 2\|\varphi\|_{v}^{2}$. Given a point $w \in \mathbf{S}^{1}$, denote by

$$
U(w, r)=\{z \in \mathbf{D}:|z-w|<r\} \quad \text { and } \quad \tau U(w, r)=\{\tau z: z \in U(w, r)\} .
$$

We prove $\|G(\tau, \varphi)\|_{v}^{2} \leq 2\|\varphi\|_{v}^{2}$ by considering the following two cases.

Case 1: We assume that $r \in(0,1]$. Clearly, if $z \in U(w, r)$, then $|z| \in[1-r, 1]$. Since $\frac{\rho_{\mathbf{D}}^{-1}(z)}{\rho_{\mathbf{D}}^{-1}(\tau z)}$ is a non-increasing function of $|z|$ for each $\tau \in[0,1]$, we obtain

$$
\sup _{z \in U(w, r)} \frac{\rho_{\mathbf{D}}^{-3}(z)}{\rho_{\mathbf{D}}^{-3}(\tau z)} \leq \sup _{z \in U(w, r)} \frac{\rho_{\mathbf{D}}^{-1}(z)}{\rho_{\mathbf{D}}^{-1}(\tau z)} \leq \frac{1-(1-r)^{2}}{1-\tau^{2}(1-r)^{2}} .
$$


Thus,

$$
\sup _{0 \leq r \leq 1}\left\{\frac{1-\tau(1-r)}{r} \sup _{z \in U(w, r)} \frac{\rho_{\mathbf{D}}^{-3}(z)}{\rho_{\mathbf{D}}^{-3}(\tau z)}\right\} \leq \sup _{0 \leq r \leq 1} \frac{2-r}{1+\tau(1-r)}<2 .
$$

Using $\tau U(w, r) \subset U(w, 1-\tau(1-r))$, we obtain

$$
\begin{aligned}
& \sup _{w, r \leq 1} \frac{\int_{U(w, r)}|G(\tau, \varphi)|^{2} \rho_{\mathbf{D}}^{-3}(z) d x d y}{r} \\
& =\sup _{w, r \leq 1} \frac{\int_{U(w, r)} \tau^{4}|\varphi(\tau z)|^{2} \rho_{\mathbf{D}}^{-3}(z) d x d y}{r} \\
& \leq \sup _{w, r \leq 1}\left\{\frac{\int_{U(w, r)} \tau^{2}|\varphi(\tau z)|^{2} \rho_{\mathbf{D}}^{-3}(\tau z) d x d y}{r} \cdot \sup _{z \in U(w, r)} \frac{\rho_{\mathbf{D}}^{-3}(z)}{\rho_{\mathbf{D}}^{-3}(\tau z)}\right\} \\
& \leq \sup _{w, r \leq 1}\left\{\frac{\int_{U(w, 1-\tau(1-r))}|\varphi(z)|^{2} \rho_{\mathbf{D}}^{-3}(z) d x d y}{1-\tau(1-r)} \cdot \frac{1-\tau(1-r)}{r} \sup _{z \in U(w, r)} \frac{\rho_{\mathbf{D}}^{-3}(z)}{\rho_{\mathbf{D}}^{-3}(\tau z)}\right\} \\
& \leq \sup _{w, r \leq 1} \frac{\int_{U(w, 1-\tau(1-r))}|\varphi(z)|^{2} \rho_{\mathbf{D}}^{-3}(z) d x d y}{1-\tau(1-r)} \cdot \sup _{r \leq 1}\left\{\frac{1-\tau(1-r)}{r} \sup _{z \in U(w, r)} \frac{\rho_{\mathbf{D}}^{-3}(z)}{\rho_{\mathbf{D}}^{-3}(\tau z)}\right\} \\
& \leq 2\|\varphi\|_{v}^{2} .
\end{aligned}
$$

Case 2: We assume that $r \in(1,2]$. Using $\tau U(w, r) \subset U(w, r)$, we obtain

$$
\begin{aligned}
\sup _{w, r \in(1,2]} \frac{\int_{U(w, r)}|G(\tau, \varphi)|^{2} \rho_{\mathbf{D}}^{-3}(z) d x d y}{r} & =\sup _{w, r \in(1,2]} \frac{\int_{U(w, r)} \tau^{4}|\varphi(\tau z)|^{2} \rho_{\mathbf{D}}^{-3}(z) d x d y}{r} \\
& \leq \sup _{w, r \in(1,2]} \frac{\int_{U(w, r)} \tau^{2}|\varphi(\tau z)|^{2} \rho_{\mathbf{D}}^{-3}(\tau z) d x d y}{r} \\
& =\sup _{w, r \in(1,2]} \frac{\int_{\tau U(w, r)}|\varphi(z)|^{2} \rho_{\mathbf{D}}^{-3}(z) d x d y}{r} \\
& \leq \sup _{w, r \in(1,2]} \frac{\int_{U(w, r)}|\varphi(z)|^{2} \rho_{\mathbf{D}}^{-3}(z) d x d y}{r} \leq\|\varphi\|_{v}^{2} .
\end{aligned}
$$

Combining the above two cases, we conclude that $\|G(\tau, \varphi)\|_{v}^{2} \leq 2\|\varphi\|_{v}^{2}$ at each point $(\tau, \varphi) \in[0,1] \times \mathcal{T}_{v}(\mathbf{D})$.

Step $2^{\prime}$. We prove that $G(\tau, \cdot)$ maps $\mathcal{T}_{v}(\mathbf{D})$ to itself for each fixed $\tau \in[0,1]$. When $\tau=1, G(\tau, \cdot)$ is the identity map and hence it maps $\mathcal{T}_{v}(\mathbf{D})$ to itself. In the following, we assume $\tau \in[0,1)$.

From Step $1^{\prime}, G(\tau, \varphi)$ is a Carleson measure on $\mathbf{D}$ for any $(\tau, \varphi) \in[0,1] \times$ $\mathcal{T}_{v}(\mathbf{D})$. By Theorem A, in order to prove $G(\tau, \varphi)$ maps $\mathcal{T}_{v}(\mathbf{D})$ to itself for any $\tau \in$ $[0,1)$, we only need to show that $G(\tau, \varphi) \in B_{v}(\mathbf{D})$ for any $\varphi \in \mathcal{T}_{v}(\mathbf{D})$. Since $\tau \in$ $[0,1), \tau U(w, r) \subset \mathbf{D}_{\tau}=\{z:|z|<\tau\}$ for any $w \in \mathbf{S}^{1}$ and any $r$. Denote by $M=$ 

$\sup _{z \in \mathbf{D}}|\varphi(\tau z)|^{2}$. Then

$$
\begin{aligned}
\sup _{w} \frac{1}{r} \int_{U(w, r)}|G(\tau, \varphi)|^{2} \rho_{\mathbf{D}}^{-3}(z) d x d y & =\sup _{w} \frac{1}{r} \int_{U(w, r)} \tau^{4}|\varphi(\tau z)|^{2} \rho_{\mathbf{D}}^{-3}(z) d x d y \\
& \leq M \tau^{4} \frac{1}{r} \int_{U(w, r)} \rho_{\mathbf{D}}^{-3}(z) d x d y \\
& \leq M \tau^{4} \frac{1}{r} \int_{0}^{2 \pi} \int_{1-r}^{1}\left(1-t^{2}\right)^{3} t d t \rightarrow 0
\end{aligned}
$$

as $r \rightarrow 0$. Hence $G(\tau, \varphi) \in B_{v}(\mathbf{D})$, which means that $G(\tau, \varphi)$ maps $\mathcal{T}_{v}(\mathbf{D})$ to itself for each $\tau \in[0,1)$.

Step $3^{\prime}$. We prove that $G:[0,1] \times \mathcal{T}_{v}(\mathbf{D}) \rightarrow \mathcal{T}_{v}(\mathbf{D})$ is continuous. Let $\varphi, \psi \in$ $\mathcal{T}_{v}(\mathbf{D})$. Using similar approaches in Step $1^{\prime}$, we obtain

$$
\|G(\tau, \varphi)-G(\tau, \psi)\|_{v}^{2} \leq 2\|\varphi-\psi\|_{v}^{2} .
$$

This inequality implies that $G(\tau, \varphi)$ is continuous on the variable $\varphi$ for each fixed for $\tau \in[0,1]$.

Now we prove that $G(\tau, \varphi)$ is continuous on the variable $\tau \in[0,1]$ for each fixed $\varphi \in \mathcal{T}_{v}(\mathbf{D})$. We divide the proof into two cases.

Case 1: Assume that a sequence $\left\{\tau_{n}\right\}_{n=1}^{\infty}$ of points in $[0,1]$ converges to a point $\tau \in[0,1)$. Then for any sufficiently large $n, \tau_{n} U(w, r)$ and $\tau U(w, r)$ are contained in a compact subset of $\mathbf{D}$ for all $w \in \mathbf{S}^{1}$ and $r$, and hence

$$
\sup _{w, r} \sup _{z \in U(w, r)}\left|G\left(\tau_{n}, \varphi\right)-G(\tau, \varphi)\right|^{2} \rightarrow 0 \text { as } n \rightarrow \infty .
$$

Since $\sup _{w, r} \frac{1}{r} \int_{U(w, r)} \rho_{\mathbf{D}}^{-3}(z) d x d y<\infty$, it is easy to see that

$$
\sup _{w, r} \frac{1}{r} \int_{U(w, r)}\left|G\left(\tau_{n}, \varphi\right)-G(\tau, \varphi)\right|^{2} \rho_{\mathbf{D}}^{-3}(z) d x d y \rightarrow 0 \text { as } n \rightarrow \infty .
$$

This means that $G(\tau, \varphi)$ is continuous at any point $\tau \in[0,1)$ for each fixed $\varphi \in \mathcal{T}_{v}(\mathbf{D})$.

Case 2: Assume that a sequence $\left\{\tau_{n}\right\}_{n=1}^{\infty}$ of points in $[0,1]$ converges to 1 . Let $\varphi \in \mathcal{T}_{v}(\mathbf{D})$. Given $\epsilon>0$, we choose $0<r_{1}<1$ such that for any $0<r \leq r_{1}$,

$$
\sup _{w \in \mathbf{S}^{1}} \frac{1}{r} \int_{U(w, r)}|\varphi(z)|^{2} \rho_{\mathbf{D}}^{-3}(z) d x d y<\epsilon .
$$

Since $\tau_{n} \rightarrow 1$, we can choose $0<r_{2}<r_{1}$ and $N \in \mathbf{N}$ such that for any $n>N$ and any $0<r \leq r_{2}$,

$$
1-\tau_{n}(1-r)<r_{1}
$$

Using similar techniques in (2.2), we obtain that for any $n>N$ and any $0<r \leq r_{2}$,

$$
\sup _{w \in \mathbf{S}^{1}} \frac{1}{r} \int_{U(w, r)}\left|G\left(\tau_{n}, \varphi\right)\right|^{2} \rho_{\mathbf{D}}^{-3}(z) d x d y<2 \epsilon .
$$
imply

Let $r_{0}=\min \left\{r_{1}, r_{2}\right\}$. When $0<r \leq r_{0}$ and $n>N$, (2.3) and (2.4) together

$$
\begin{aligned}
& \sup _{w \in \mathbf{S}^{1}} \frac{1}{r} \int_{U(w, r)}\left|G\left(\tau_{n}, \varphi\right)-\varphi(z)\right|^{2} \rho_{\mathbf{D}}^{-3}(z) d x d y \\
& \leq \sup _{w \in \mathbf{S}^{1}} \frac{2}{r} \int_{U(w, r)}\left(\left|G\left(\tau_{n}, \varphi\right)\right|^{2}+|\varphi(z)|^{2}\right) \rho_{\mathbf{D}}^{-3}(z) d x d y<6 \epsilon .
\end{aligned}
$$


Now we assume $r>r_{0}$. Given a point $w \in \mathbf{S}^{1}$, we denote by $\widehat{w^{*} w^{* *}}$ the piece of the boundary of $U(w, r)$ on $\mathbf{S}^{1}$, where $w^{*}, w^{* *}$ are the endpoints of $\widehat{w^{*} w^{* *}}$. We choose $w_{1}, w_{2}, \cdots, w_{k}$ on $\mathbf{S}^{1}$ satisfying that $w_{1}=w^{*},\left|w_{i}-w_{i+1}\right|=r_{0}$ for any $1 \leq i<i+1 \leq$ $k$, and $w^{* *} \in \bar{U}\left(w_{k}, r_{0}\right)$ but $w^{* *} \notin \bar{U}\left(w_{i}, r_{0}\right)$ for $1 \leq i \leq k-1$. It is obvious that

$$
k \leq\left[\frac{2 r}{r_{0}}\right]+1
$$

where $\left[\frac{2 r}{r_{0}}\right]$ denotes the integer part of $\frac{2 r}{r_{0}}$. Denote by $w_{k+1}=w^{* *}$ and $V(w, r)=$ $U(w, r) \backslash \cup_{i=1}^{k+1} \overline{U\left(w_{i}, r_{0}\right)}$. Then $V(w, r)$ is contained in a compact set of $\mathbf{D}$ for all $w \in \mathbf{S}^{1}$ and $r>r_{0}$. Hence for the given $\epsilon>0$, we can choose $N^{\prime} \in \mathbf{N}$ such that for any $n>N^{\prime}$,

$$
\sup _{z \in V(w, r)}\left|G\left(\tau_{n}, \varphi\right)-\varphi(z)\right|^{2}<\epsilon r_{0} .
$$

Using (2.5) and (2.6) and the construction of $w_{1}, w_{2}, \cdots, w_{k}$, we obtain for any $n>$ $\max \left\{N, N^{\prime}\right\}$,

$$
\begin{aligned}
& \sup _{w \in \mathbf{S}^{1}} \frac{1}{r} \int_{U(w, r)}\left|G\left(\tau_{n}, \varphi\right)-\varphi(z)\right|^{2} \rho_{\mathbf{D}}^{-3}(z) d x d y \\
& \leq \sup _{w \in \mathbf{S}^{1}} \frac{1}{r} \int_{V(w, r)}\left|G\left(\tau_{n}, \varphi\right)-\varphi(z)\right|^{2} \rho_{\mathbf{D}}^{-3}(z) d x d y \\
& \quad+\sup _{w \in \mathbf{S}^{1}} \frac{r_{0}}{r} \sum_{i=1}^{k+1} \frac{1}{r_{0}} \int_{U\left(w_{i}, r_{0}\right)}\left|G\left(\tau_{n}, \varphi\right)-\varphi(z)\right|^{2} \rho_{\mathbf{D}}^{-3}(z) d x d y \\
& \leq \epsilon \frac{r_{0}}{r} \int_{\mathbf{D}} \rho_{\mathbf{D}}^{-3}(z) d x d y+\frac{r_{0}}{r}(k+1)(6 \epsilon) \leq \frac{\pi}{2} \epsilon+6 \frac{2 r+2 r_{0}}{r} \epsilon<26 \epsilon .
\end{aligned}
$$

By (2.5) and (2.7), we conclude that

$$
\left\|G\left(\tau_{n}, \varphi\right)-G(1, \varphi)\right\|_{v}^{2} \rightarrow 0 \text { as } n \rightarrow \infty .
$$

This means $G(\tau, \varphi)$ is continuous at $\tau=1$ for each fixed $\varphi \in \mathcal{T}_{v}(\mathbf{D})$.

By considering the above two cases, we have shown that $G(\tau, \varphi)$ is continuous on the variable $\tau \in[0,1]$ for each fixed $\varphi \in \mathcal{T}_{v}(\mathbf{D})$.

Step $4^{\prime}$. We prove that $G(\tau, \cdot): \mathcal{T}_{v}(\mathbf{D}) \rightarrow B_{v}(\mathbf{D})$ is holomorphic for each fixed $\tau \in[0,1]$. Using the fact that $A=\left\{l_{z}: z \in \mathbf{D}^{*}\right\}$ is also a total subset of the dual space $B_{v}^{*}(\mathbf{D})$, the proof of this step is identical to the one of Step 3.

Combining Steps $1^{\prime}-4^{\prime}$, we conclude that $G(\tau, \varphi)$ is continuous on both variables and it maps $\mathcal{T}_{v}(\mathbf{D})$ holomorphically to itself for each fixed $\tau \in[0,1]$.

\section{Proofs of Theorem 3 and Corollary 1}

It is given in [28] that the Kobayashi metric coincides with the Teichmüller metric on the Weil-Petersson Teichmüller space $T_{2}(\mathbf{D})$. In addition, a criterion (see the following Theorem B) is given in [28] for a subspace of $T(\mathbf{D})$ to have the two metrics to be same. In this section we use that criterion to show our Theorem 3, although we first obtained this result through a quite direct approach (see [11]).

Theorem B. [28] Let $T^{\prime}$ be a complex manifold with a holomorphic embedding $\iota$ of $T^{\prime}$ into $T(\mathbf{D})$, and identify $T^{\prime}$ with $\iota\left(T^{\prime}\right)$. If $T^{\prime}$ satisfies the following three conditions, then the Teichmüller distance on $T^{\prime}$ coincides with the Kobayashi distance.

(1) The set $T^{\prime} \backslash[0]$ is contained in the set of Strebel points of $T(\mathbf{D})$; 
(2) For any $\tau \in T^{\prime}$, the right translation map for $\tau$ maps $T^{\prime}$ onto itself;

(3) For every $\tau \in T^{\prime} \backslash[0]$, there exists a representative $\mu \in \tau$ corresponding to a frame mapping such that, for every $\mu^{\prime} \in \tau$ that coincides with $\mu$ outside some compact subset of $\mathbf{D}$ and for every $t \in \mathbf{D},\left[t \mu^{\prime}\right] \in T^{\prime}$.

A Strebel point of $T(\mathbf{D})$ is a point $[\mu]$ of $T(\mathbf{D})$ containing a frame mapping, which is a quasiconformal homeomorphism of $\mathbf{D}$ whose maximal dilatation on the complement of some compact subset of $\mathbf{D}$ is strictly less than the extremal maximal dilatation of $[\mu]$. For more information on Strebel points, we refer to [17], [25] and [13].

Proof of Theorem 3. Using Theorem 1 and the facts that $T_{v}(\mathbf{D}) \subset T_{0}(\mathbf{D})$ and $T_{v}(\mathbf{D})$ is a group, it suffices to prove that $T_{v}(\mathbf{D})$ satisfies the condition (3) in Theorem B. For this purpose, we only need to show for any point $\tau \in T_{v}(\mathbf{D})$, there exists a representative $\mu$ of $\tau$ such that $\mu \in M_{v}(\mathbf{D}) \cap M_{0}(\mathbf{D})$. In the following, we prove the existence of such a representative $\mu$ for $\tau$. By the definition of $T_{v}(\mathbf{D})$, it is easy to check that the constructed $\mu$ satisfies the condition (3).

For any $\tau=[\nu] \in T_{v}(\mathbf{D})$, let $h=\left.f^{\nu}\right|_{\mathbf{S}^{1}}$ and $e x\left(h^{-1}\right)$ be the Douady-Earle

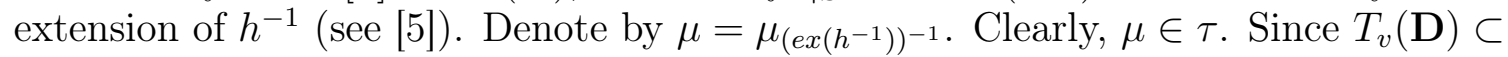
$T_{0}(\mathbf{D}),[\mu] \in T_{0}(\mathbf{D})$. It follows from Theorem 4 of [10] that $\mu_{e x(h)} \in M_{0}(\mathbf{D})$. Then $\mu_{(e x(h))^{-1}} \in M_{0}(\mathbf{D})$ and hence $\left[h^{-1}\right] \in T_{0}(\mathbf{D})$. Again by Theorem 4 of [10], we know $\mu_{e x\left(h^{-1}\right)} \in M_{0}(\mathbf{D})$, hence $\mu \in M_{0}(\mathbf{D})$.

To prove $\mu \in M_{v}(\mathbf{D})$, we recall two results from [23] and [4]. Keep in mind that $C M_{0}(\mathbf{D})$ denotes the collection of vanishing Carleson measures on $\mathbf{D}$.

Lemma 3. [23] Assume that $\alpha, \beta>0$. For a positive measure $\lambda$ on $\mathbf{D}$, let

$$
\tilde{\lambda}(z)=\int_{\mathbf{D}} \frac{\left(1-|z|^{2}\right)^{\alpha}\left(1-|w|^{2}\right)^{\beta}}{|1-\bar{z} w|^{\alpha+\beta+2}} \lambda(w) d u d v, \quad \text { where } w=u+i v .
$$

Then $\tilde{\lambda} \in C M_{0}(\mathbf{D})$ if $\lambda \in C M_{0}(\mathbf{D})$.

Lemma 4. [4] Let $\tilde{h}$ be a quasisymmetric homeomorphism of $\mathbf{S}^{1}$. There exists a constant $C$ depending on the ratio distortion norm of $\tilde{h}$ such that $\forall z \in \mathbf{D}$,

$$
\frac{\left|\mu_{f^{-1}}(z)\right|^{2}}{1-\left|\mu_{f^{-1}}(z)\right|^{2}} \leq C \int_{\mathbf{D}} \frac{\left|\mu_{g^{-1}}(w)\right|^{2}}{1-\left|\mu_{g^{-1}}(w)\right|^{2}} \frac{(1-|z|)^{2}}{|1-\bar{z} w|^{4}} d u d v
$$

where $w=u+i v, f$ is the Douady-Earle extension of $\tilde{h}$ and $g$ is a quasiconformal extension of $\tilde{h}$. that

Since $\tau=[h]=[\nu] \in T_{v}(\mathbf{D})$, there exists a quasiconformal extension $\tilde{g}$ of $h$ such

$$
\left|\mu_{\tilde{g}}(w)\right|^{2} \rho_{\mathbf{D}}(w) \in C M_{0}(\mathbf{D}) .
$$

Let $g=\tilde{g}^{-1}$. Then $g$ is a quasiconformal extension of $h^{-1}$ satisfying

$$
\left|\mu_{g^{-1}}(w)\right|^{2} \rho_{\mathbf{D}}(w) \in C M_{0}(\mathbf{D}) .
$$

Applying Lemma 3 to the case when $\alpha=1, \beta=1$ and $\lambda(w)=\left|\mu_{g^{-1}(w)}\right|^{2} \rho_{\mathbf{D}}(w)$, we obtain

$$
\int_{\mathbf{D}}\left|\mu_{g^{-1}}(w)\right|^{2} \frac{1-|z|^{2}}{|1-\bar{z} w|^{4}} d u d v \in C M_{0}(\mathbf{D}) .
$$

Since $g^{-1}$ is a quasiconformal map, the previous condition implies

$$
\int_{\mathbf{D}} \frac{\left|\mu_{g^{-1}}(w)\right|^{2}}{1-\left|\mu_{g^{-1}}(w)\right|^{2}} \frac{1-|z|^{2}}{|1-\bar{z} w|^{4}} d u d v \in C M_{0}(\mathbf{D})
$$


which is equal to

$$
\frac{1+|z|}{1-|z|} \int_{\mathbf{D}} \frac{\left|\mu_{g^{-1}}(w)\right|^{2}}{1-\left|\mu_{g^{-1}}(w)\right|^{2}} \frac{(1-|z|)^{2}}{|1-\bar{z} w|^{4}} d u d v \in C M_{0}(\mathbf{D})
$$

Combined with applying Lemma 4 to $\tilde{h}=h^{-1}, f=e x\left(h^{-1}\right)$ and $g$, we conclude

$$
\frac{1+|z|}{1-|z|} \frac{\left|\mu_{f^{-1}}(z)\right|^{2}}{1-\left|\mu_{f^{-1}}(z)\right|^{2}} \in C M_{0}(\mathbf{D}) .
$$

Since $f^{-1}$ is a quasiconformal map, it follows that

$$
\left|\mu_{f^{-1}}(z)\right|^{2} \rho_{\mathbf{D}}(z) \in C M_{0}(\mathbf{D}) \text {, i.e., } \mu_{f^{-1}} \in M_{v}(\mathbf{D}) .
$$

Therefore,

$$
\mu \in M_{v}(\mathbf{D}) \cap M_{0}(\mathbf{D}) .
$$

Before giving a proof to Corollary 1, we introduce a result of [18].

Lemma 5. [18] Let $d_{C, T(\mathbf{D})}$ and $d_{K, T(\mathbf{D})}$ be the Carathéodory metric and the Kobayashi metric on $T(\mathbf{D})$ respectively. Then, for any $p, q \in T(\mathbf{D})$, there is a universal constant $\alpha \geq 1$ such that

$$
d_{C, T(\mathbf{D})}(p, q) \leq d_{K, T(\mathbf{D})}(p, q) \leq \alpha d_{C, T(\mathbf{D})}(p, q)
$$

Applying Theorems 1 and 3 to $T_{2}(\mathbf{D})$ and $T_{v}(\mathbf{D})$, we obtain a similar result to Lemma 5.

Lemma 6. Let $d_{C, T_{2}}$ and $d_{K, T_{2}}$ be the Carathéodory metric and Kobayashi metric on $T_{2}(\mathbf{D})$ respectively, let $d_{C, T_{v}}$ and $d_{K, T_{v}}$ be the Carathéodory metric and the Kobayashi metric on $T_{v}(\mathbf{D})$ respectively, and let $\alpha$ be the same constant as in Lemma 5. Then for any two points $p, q \in T_{2}(\mathbf{D})$,

$$
d_{C, T_{2}}(p, q) \leq d_{K, T_{2}}(p, q) \leq \alpha d_{C, T_{2}}(p, q)
$$

for any two points $p, q \in T_{v}(\mathbf{D})$,

$$
d_{C, T_{v}}(p, q) \leq d_{K, T_{v}}(p, q) \leq \alpha d_{C, T_{v}}(p, q) .
$$

Proof. Since $T_{2}(\mathbf{D})$ is a complex Banach manifold, for any $p, q \in T_{2}(\mathbf{D})$ the inequality $d_{C, T_{2}}(p, q) \leq d_{K, T_{2}}(p, q)$ holds. Using Theorem 1, we obtain

$$
d_{C, T_{2}}(p, q) \geq d_{C, T(\mathbf{D})}(p, q) .
$$

It follows from Lemma 5 and Theorem 3 that

$$
d_{C, T(\mathbf{D})}(p, q) \geq \frac{1}{\alpha} d_{K, T(\mathbf{D})}(p, q)=\frac{1}{\alpha} d_{T}(p, q)=\frac{1}{\alpha} d_{K, T_{2}}(p, q) .
$$

Hence, the double inequality (3.2) holds.

Using the same argument, we conclude the double inequality (3.3).

Proof of Corollary 1. Let $\mu \in M_{0}(\mathbf{D})$ and $[\mu] \in T_{0}(\mathbf{D}) \backslash T_{2}(\mathbf{D})$, and let $\left\{r_{n}\right\}_{n=1}^{\infty}$ be an increasing sequence of positive real numbers converging to 1 . We set

$$
\mu_{n}(z)= \begin{cases}\mu(z), & z \in \mathbf{D}_{r_{n}}, \\ 0, & z \in \mathbf{D}_{r_{n}, 1} .\end{cases}
$$

Then $\left\{\left[\mu_{n}\right]\right\}_{n=1}^{\infty} \subset T_{2}(\mathbf{D})$ is a Cauchy sequence in $T_{2}(\mathbf{D})$ under the metric $d_{T_{2}}=d_{K, T_{2}}$ and furthermore the limit of $\left\{\left[\mu_{n}\right]\right\}_{n=1}^{\infty}$ is $[\mu]$.

Let $d$ be an invariant metric on $T_{2}(\mathbf{D})$. For any two points $p, q \in T_{2}(\mathbf{D})$, it is clear that

$$
d_{C, T_{2}}(p, q) \leq d(p, q) \leq d_{K, T_{2}}(p, q)
$$


By Lemma 6,

$$
\frac{1}{\alpha} d_{K, T_{2}}(p, q) \leq d_{C, T_{2}}(p, q) \leq d(p, q) \leq d_{K, T_{2}}(p, q) .
$$

Thus, $\left\{\left[\mu_{n}\right]\right\}_{n=1}^{\infty}$ is a Cauchy sequence under the metric $d$. Suppose that $\left[\mu^{*}\right]$ is a limit of this sequence in $T_{2}(D)$ under the metric $d$. Using the previous inequality, we know that $\left[\mu^{*}\right]$ is also a limit of $\left\{\left[\mu_{n}\right]\right\}_{n=1}^{\infty}$ under the Teichmüller metric on $T_{2}(\mathbf{D})$ and hence on $T(\mathbf{D})$. By the uniqueness of limits, $[\mu]=\left[\mu^{*}\right]$. This is a contradiction. Therefore, the sequence $\left\{\left[\mu_{n}\right]\right\}_{n=1}^{\infty}$ has no limit in $T_{2}(\mathbf{D})$ under the metric $d$. Thus, $T_{2}(\mathbf{D})$ is not complete under the metric $d$.

Using a similar argument, we conclude that $T_{v}(\mathbf{D})$ is not complete under any invariant metric $d$ too.

\section{References}

[1] Astala, K., and M. Zinsmeister: Teichmüller spaces and BMOA. - Math. Ann. 289, 1991, 613-625.

[2] Bishop, C., and P. Jones: Harmonic measure, $L^{2}$ estimates and the Schwarzian derivative. - J. Anal. Math. 62, 1994, 77-113.

[3] CUI, G.: Integrably asymptotic affine homeomorphisms of the circle and Teichmüller spaces. Sci. China Ser. A 43, 2000, 267-279.

[4] Cui, G., and M. Zinsmeister: BMO-Teichmüller spaces. - Illinois J. Math. 48, 2004, 12231233.

[5] Douady, A., and C. Earle: Conformally natural extension of homeomorphisms of the circle. - Acta Math. 157, 1986, 23-48.

[6] Earle, C. J.: The holomorphic contractibility of two generalized Teichmüller spaces. - Publ. Inst. Math. 75:89, 2004, 109-117.

[7] Earle, C. J., F. P. Gardiner, and N. Lakic: Asymptotic Teichmüller space. I. The complex structure. - In: In the tradition of Ahlfors and Bers, Contemp. Math. 256, Amer. Math. Soc., Providence, RI, 2000, 17-38.

[8] Earle, C. J., F. P. Gardiner, and N. Lakic: Asymptotic Teichmüller space. II. The metric structure. - In: In the tradition of Ahlfors and Bers III, Contemp. Math. 355, Amer. Math. Soc., Providence, RI, 2004, 187-219.

[9] Earle, C. J., I. Kra, and S. L. Krushkal: Holomorphic motions and Teichmüller spaces. Trans. Amer. Math. Soc. 343, 1994, 927-948.

[10] Earle, C. J., V. Markovic, and D. Šarić: Barycentric extension and the Bers embedding for asymptotic Teichmüller space. - In: Complex manifolds and hyperbolic geometry, 87-105, Contemp. Math. 311, Amer. Math. Soc., Providence, RI, 2002.

[11] FAN, J., and J. Hu: A gluing theorem and applications to subspaces of the universal Teichmüller space. - Preprint, 2013.

[12] Gardiner, F. P.: Approximation of infinite-dimensional Teichmüller spaces. - Trans. Amer. Math. Soc. 282, 1984, 367-383.

[13] Gardiner, F.P., and N. LakiC: Quasiconformal Teichmüller spaces. - Math. Surveys Monogr. 76, Amer. Math. Soc., Providence, 2000.

[14] Gardiner, F. P., and D. Sullivan: Symmetric structures on a closed curve. - Amer. J. Math. 114, 1992, 683-736.

[15] Hu, J., Y. JiAnG, and Z. WANG: Kobayashi's and Teichmüller's metrics on the Teichmüller space of symmetric circle homeomorphisms. - Acta Math. Sin. (Engl. Ser.) 27, 2011, 617-624.

[16] Hu, Y., and Y. Shen: On quasisymmetric homeomorphisms. - Israel J. Math. 191, 2012, 209-226. 
[17] Lakic, N.: Strebel points. - Contemp. Math. 211, 1997, 417-431.

[18] Miүachi, H.: On invariant distances on asymptotic Teichmüller spaces. - Proc. Amer. Math. Soc. 134, 2006, 1917-1925.

[19] NAG, S.: The Complex analytic theory of Teichmüller spaces. - Wiley-Interscience, 1988.

[20] Pommerenke, Сh.: On univalent functions, Bloch functions and VMOA. - Math. Ann. 236, 1978, 199-208.

[21] Royden, H. L.: Automorphisms and isometries of Teichmüller space. - In: Advances in the Theory of Riemann Surfaces, Ann. of Math. Studies, No. 66. 369-383, Princeton Univ. Press, Princeton, 1971.

[22] Shen, Y.: Weil-Petersson Teichmüller space. - arXiv:1304.3197.

[23] Shen, Y., and H. Wer: Universal Teichmüller space and BMO. - Adv. Math. 234, 2013, $129-148$.

[24] Shen, Y.: On Grunsky operator. - Sci. China Ser. A 50, 2007, 1805-1817.

[25] Strebel, K.: On the existence of extremal Teichmüller mappings. - J. Anal. Math. 30, 1976, $464-480$.

[26] Takhtajan, L., and L. Teo: Weil-Petersson metric on the universal Teichmüller space. Mem. Amer. Math. Soc. 183:861, 2006.

[27] TAng, S., H. Wei, and Y. Shen: VMOA-Teichmüller space is contractible. - Preprint, 2015.

[28] Yanagishita, M.: Teichmüller distance and Kobayashi distance on subspaces of the universal Teichmüller space. - Kodai Math. J. 36, 2013, 209-227.

Received 17 September 2015 • Accepted 13 November 2015 